\title{
$A b$ initio validation on the connection between atomistic and hydrodynamic description to unravel the ion dynamics of warm dense matter
}

\author{
Qiyu Zeng, ${ }^{1}$ Xiaoxiang Yu $\odot,{ }^{1}$ Yunpeng Yao, ${ }^{1}$ Tianyu Gao, ${ }^{1}$ Bo Chen, ${ }^{1}$ Shen Zhang, ${ }^{1}$ Dongdong Kang, ${ }^{1}$ Han Wang, ${ }^{2}$ \\ and Jiayu Dai $\oplus^{1, *}$ \\ ${ }^{1}$ Department of Physics, National University of Defense Technology, Changsha, Hunan 410073, People's Republic of China \\ ${ }^{2}$ Laboratory of Computational Physics, Institute of Applied Physics and Computational Mathematics, \\ Beijing 100088, People's Republic of China
}

(Received 12 January 2021; revised 21 April 2021; accepted 19 July 2021; published 5 August 2021)

\begin{abstract}
Ion dynamics exhibits inherent multiscale characteristics because it contains both atomistic and hydrodynamic behaviors. Although atomic-scale ab initio molecular dynamics is the subject of intense research on warm dense matter, the macroscopic relaxation process contained in the zero-frequency mode of the ionic dynamic structure factor (DSF) cannot be demonstrated due to the limitation of simulation sizes. Here, we fill this gap via the machine-learning deep potential method. To capture the ion dynamics near the hydrodynamic limit with $a b$ initio accuracy, an accurate and efficient electron-temperature-dependent interatomic potential was constructed. We quantitatively verify the consistency of thermal diffusivities obtained from hydrodynamics and the fluctuationdissipation theorem and further provide a microscopic perspective of energy transport to understand the zerofrequency mode of DSF. As implemented in two temperature states, a competitive mechanism is found to account for the damping of the zero-frequency mode.
\end{abstract}

DOI: 10.1103/PhysRevResearch.3.033116

\section{INTRODUCTION}

The ion-ion dynamic structure factor (DSF), known as the power spectrum of the reciprocal-space Van Hove function (density-density time correlation function) [1], describes the relaxation and propagation processes spanning different spatial scales. DSF plays a key role in the study of ion dynamics, such as phase transition [2,3], collective modes [4,5], dissipation processes [6], etc. There are two perspectives at different scales to obtain and understand the DSF. At the atomistic scale, the microscopic behaviors can be captured by recording the trajectories in molecular dynamics (MD) simulations [7-9] or revealed experimentally by the inelastic scattering of radiation or a particle whose wavelength is comparable to the interparticle spacing [10-12]. Hence, for a system the DSF gives its response to density fluctuations of frequency $\omega$ and wave vector $\mathbf{k}$ and is defined as

$$
\begin{aligned}
S(\mathbf{k}, \omega) & =\frac{1}{2 \pi} \int_{-\infty}^{\infty} \frac{1}{N}\langle\rho(\mathbf{k}, t) \rho(-\mathbf{k}, 0)\rangle e^{i \omega t} d t \\
& =\frac{1}{2 \pi N T}|\rho(\mathbf{k}, \omega)|^{2},
\end{aligned}
$$

where $N$ is the number of ions and the brackets represent the ensemble average; the quantity $\rho(\mathbf{k}, t)=\sum_{i=1}^{N} \exp [-i \mathbf{k}$.

\footnotetext{
*jydai@ nudt.edu.cn

Published by the American Physical Society under the terms of the Creative Commons Attribution 4.0 International license. Further distribution of this work must maintain attribution to the author(s) and the published article's title, journal citation, and DOI.
}

$\left.\mathbf{r}_{i}(t)\right]$ is the Fourier transform of the time-dependent ion density $\rho(\mathbf{r}, t)$ in real space for a period of $T$.

At the macroscopic scale, only the macroscopic processes can survive and contribute to the DSF [1]. Within the framework of classical hydrodynamics, it demonstrates that the DSF consists of three eigenmodes, expressed in terms of phenomenological macroscopic transport coefficients, namely, the two sound waves propagating in opposite directions and one relaxing thermally diffusive mode, defined as

$$
\begin{aligned}
S(k, \omega)= & \frac{S(k)}{2 \pi}\left[\left(\frac{\gamma-1}{\gamma}\right) \frac{2 D_{T} k^{2}}{\omega^{2}+\left(D_{T} k^{2}\right)^{2}}\right. \\
& +\frac{1}{\gamma}\left(\frac{\Gamma k^{2}}{\left(\omega+c_{s} k^{2}\right)^{2}+\left(\Gamma k^{2}\right)^{2}}\right. \\
& \left.\left.+\frac{\Gamma k^{2}}{\left(\omega-c_{s} k^{2}\right)^{2}+\left(\Gamma k^{2}\right)^{2}}\right)\right],
\end{aligned}
$$

where $D_{T}=\kappa / \rho c_{p}$ is ionic thermal diffusivity, with $\kappa$ being the ionic thermal conductivity. $c_{S}$ is the adiabatic speed of sound, the sound attenuation constant $\Gamma=(\gamma-1) D_{T} / 2+$ $b / 2$, where $b$ is the kinematic longitudinal viscosity, and $\gamma=$ $c_{p} / c_{V}$ is the ratio of the heat capacity at constant pressure $c_{p}$ to the heat capacity at constant volume $c_{V}$.

The molecular dynamics simulations contain the atomic thermal motion and interatomic interaction, which directly captures the complex contribution to DSF from first principles. By contrast, the hydrodynamic description based on the continuum assumption enables us to analytically derive the DSF via the macroscopic transport coefficients. However, there is a huge gap between the atomistic and hydrodynamic descriptions. We should note that the hydrodynamic description is valid only at a scale of length and time much larger 
than the atomistic level. But the probing scale available in microscopic techniques, such as ab initio MD simulations and inelastic scattering experiments, is still far from the hydrodynamic limit. According to Eqs. (1) and (2), it seems that the theoretical bridge between the microscopic and macroscopic descriptions has been established. However, we did not know at which spatial scales they are consistent, and quantitative validation of the consistency of the microscopic quantities and macroscopic transport coefficients remains absent. Some issues, such as how to verify the hydrodynamic description through atomistic approaches, how the physical quantities evolve when approaching the hydrodynamic limit, etc., have not been clarified. Should such a multiscale framework be established, it will directly point out a new solution to obtain transport properties (thermal conductivity, viscosity, and the diffusion coefficient) by ion-ion DSF at small $k$ values from simulations or experiments.

Understanding the ion dynamics of warm dense matter (WDM) is one of the central points for many physical systems such as the interior of giant planets [13], laser machining and ablation [14], and inertial-confinement fusion [15]. The disordered system, consisting of strongly coupled classical ions that coexist with partially or fully degenerate electrons [16], introduces huge difficulties to describing the ion dynamics accurately.

In recent decades, tremendous efforts have been made to extract the DSF of WDM based on numerical simulations $[6-9,17,18]$. Most of them have focused on the non-zerofrequency mode at the microscopic scale, i.e., the acoustic collective excitations and dispersion relation [17,19,20]. Except for the acoustic collective modes, special attention had been paid to the distinct effects on DSF. The nonadiabatic effect leads to a strongly enhanced zero-frequency mode (ZFM) [6], while significant damping of the ZFM and hardening of the acoustic collective mode can be observed under two temperature states [18]. However, a clear demonstration of the microscopic mechanism behind these phenomena is still lacking, especially for the behavior of the ZFM. The ZFM represents relaxation processes that dominate the temporal decay of the density fluctuation. As discussed above, the underlying connection between the microscopic dynamics and the macroscopic transport coefficients can provide a new path to extract the basic physics contained in the ZFM.

In addition, despite the great experimental progress, it is still quite challenging to make a long-lived sample of WDM [21]. Therefore, the experimental characterization of transport coefficients remains lacking for WDM. Since the ion-ion DSF can be measured directly or indirectly by inelastic scattering experiments [10,22], it could be a critical path to probe the ion dynamics related physics [22,23]. Establishing the multiscale connection would be directly beneficial to probing the transport coefficients from experiments.

In this work, the ion dynamics from both the atomistic and hydrodynamic descriptions are presented by investigating the DSF of warm dense aluminum. The deep potential (DP) method is utilized to describe the interatomic interaction in the WDM regime, allowing us to approach the hydrodynamic limit with the accuracy of density functional theory (DFT) methods using more than 100000 atoms. Thermal diffusivity, a transport coefficient related to the energy and mass transport, serves as the key quantity in the multiscale framework to understand the ZFM. We quantitatively verify the consistency of the thermal diffusivity obtained from the hydrodynamic description and the fluctuation-dissipation theorem; the latter represents microscopic dynamics with a very large wavelength (small wave number). The nonequilibrium effect on ion dynamics is studied. We analyze the single-particle diffusional motion and collective motion, which both contribute to the ZFM in DSF, and we elucidate the mechanism accounting for the damping of ZFM from the perspectives of mass and energy transport.

\section{COMPUTATIONAL METHODS}

Considering the wide range of temperature of WDM, the electron-temperature-dependent DP method [24-27] was used to generate a many-body potential in the form of neural networks. By introducing explicit dependence of a free-energy surface on the electron temperature $T_{e}$, the electronic effect of excited states such as phonon hardening [28] can be included. We ran Born-Oppenheimer molecular dynamics (BOMD) simulations with Kohn-Sham density functional theory (KSDFT) to generate two-temperature warm dense aluminum trajectories $\left(\rho_{0}=2.7 \mathrm{~g} / \mathrm{cm}^{3}, T_{i}=1 \mathrm{eV}\right.$, and $T_{e}=1,5$, and $10 \mathrm{eV}$ ) by using the QUANTUM ESPRESSO package [29]. The Perdew-Burke-Ernzerhof (PBE) exchange-correlation (XC) functional [30] was used. Pseudopotential that takes the projector augmented-wave (PAW) formalism [31,32] is adopted with eleven valence electrons. The sampling of the Brillouin zone was carried out at the Baldereschi mean value point [33]. We adopted the deep neutral network (DNN) based models trained from BOMD trajectories with the DEEPMD-KIT package [25]. We performed all the deep potential molecular dynamics (DPMD) simulations with the LAMMPS package [34]. The time step was set to 1 fs and the Nose-Hoover thermostat [35,36] was employed in the NVT ensemble. After a thermalization of 50000 steps, the configurations were stored every 10 steps during the next 150 000-step long duration (150 ps). The generated DP can faithfully reproduce both the static and dynamic structures, as shown in Figs. S9-S13 in the Supplemental Material [37], allowing us to achieve large-scale simulations with a precise description of the interatomic effective interaction under twotemperature states. For both equilibrium and nonequilibrium warm dense aluminum, our results show good agreement with previous KS-DFT [8], orbital-free DFT (OF-DFT) [38], and the neutral pseudoatom MD (NPA-MD) results [18], as shown in Fig. S13 [37].

\section{CONSISTENCY OF HYDRODYNAMICS AND THE FLUCTUATION-DISSIPATION THEOREM}

The hydrodynamic description can yield transport properties by fitting the DSF in the form of Eq. (2) [39]. Alternatively, a different way to describe transport properties is derived from the fluctuation-dissipation theorem, to be specific, the Green-Kubo (GK) formula based on linear response theory [40-43]. To self-consistently validate the hydrodynamic description of DSF in the WDM regime, namely, 

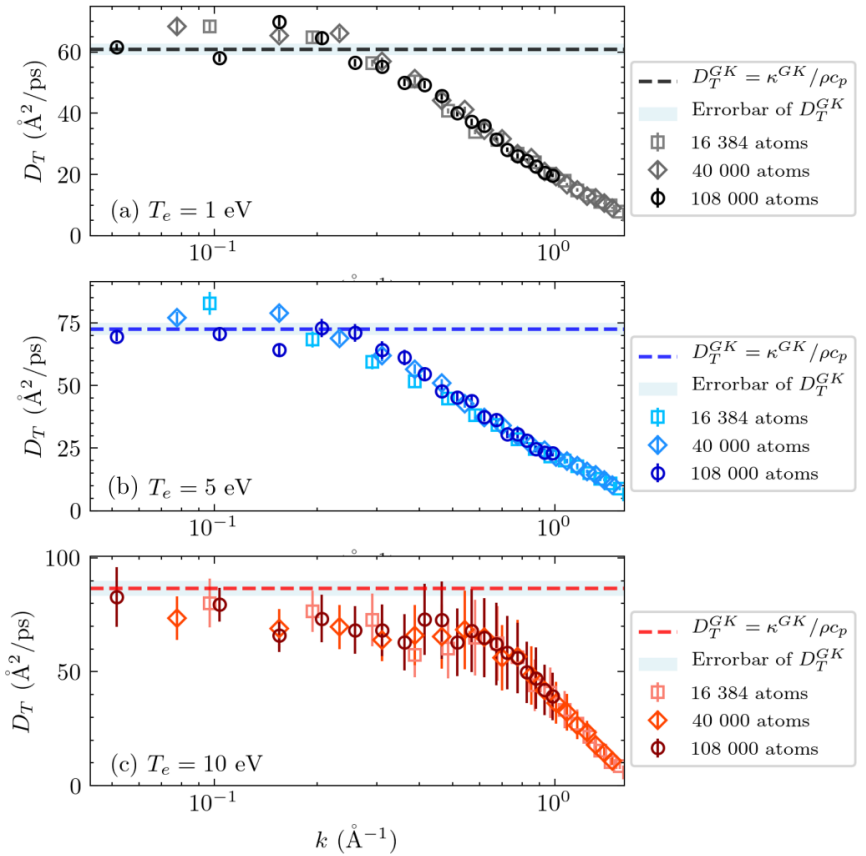

FIG. 1. Thermal diffusivities $D_{T}$ of warm dense aluminum calculated at (a) equilibrium conditions $\rho_{0}=2.7 \mathrm{~g} / \mathrm{cm}^{3}, T_{i}=T_{e}=$ $1 \mathrm{eV}$ and (b) and (c) nonequilibrium conditions $T_{i}=1 \mathrm{eV}$ and $T_{e}=5 \mathrm{eV}, 10 \mathrm{eV}$. The open squares, diamonds, and circles show the fitted value of $D_{T}^{D S F}(k)$ from the DSF calculated at the $k$ value, where $k=n \Delta k=n \frac{2 \pi}{L}, n \in \mathbf{Z} . D_{T}^{G K}=\kappa^{G K} /\left(\rho c_{p}\right)$, where $\kappa^{G K}$ obtained from the GK formula is given as the benchmark, marked with a dashed line.

whether the wave number is small enough to approach the hydrodynamic limit, thermal conductivity is selected as the key quantity.

To obtain ionic thermal conductivity $\kappa^{G K}$ from the GK relation, a series of system sizes ranging from 32 to 16384 atoms was simulated to consider the size effect $[37,44]$. We chose the converged $\kappa^{G K}$ results to obtain the thermal diffusivity $D_{T}^{G K}$ through $D_{T}=\kappa / \rho c_{p}$. This quantity serves as the benchmark to represent the intrinsic energy transport properties of the ions, as shown in Fig. 1. Also, with $D_{T}$ and $b$ as the two fitting parameters at each $k$ value, ionic thermal diffusivity $D_{T}^{D S F}$ can be obtained by fitting DSF via Eq. (2) (see the Supplemental Material [37]), as presented in Fig. 1. The DSF is calculated via Eq. (1) at 20 equally spaced $k$ values $n \Delta k(n=1,2, \ldots, 20)$, where $\Delta k=2 \pi / L$ is the smallest $k$ value that is limited by system size. A large system size ranging from 16384 to 108000 atoms was used for this purpose. This comparison of $D_{T}^{G K}$ and $D_{T}^{D S F}$ measures the validity of the hydrodynamic description at various spatial scales. The actual physical meaning of $D_{T}^{D S F}(k)$ in the intermediate-wavenumber regime remains undetermined because the thermally diffusive mode may not contribute dominantly to the ZFM beyond the hydrodynamic regime [4].

As clearly shown in Fig. 1, the thermal diffusivities fitted from DSF exhibit an obvious wave number dependence at intermediate $k$ values and gradually converges to the results calculated by the GK formula as $k$ values decrease below $0.2 \AA^{-1}$ (the system contains more than 10000 atoms correspondingly), confirming the consistency between the hydrodynamic description and fluctuation-dissipation theorem in this near-hydrodynamic regime. This quantitative validation indicates that the hydrodynamic limit can be reached in our MD simulation with a large system containing more than 16384 atoms.

Most importantly, the connection between the ZFM in DSF and energy transport is established. At the near-hydrodynamic limit, the macroscopic thermal relaxation processes dominate the ZFM, suggesting that the microscopic dynamics behind ZFM is purely ionic energy transport. The verified relationship between ZFM and energy transport establishes a path to understand the behavior of the ZFM, allowing the analysis of the microscopic mechanism of various significant effects (the nonequilibrium condition and nonadiabatic effect, for example) on the DSF. Although Mithen et al. used the dispersion relation to validate the hydrodynamic description in dense plasma [19], two key points need to be made: (1) An ab initio validation with DFT accuracy is implemented, which is highly desirable for describing the WDM compared with simple systems based on the Yukawa model [45]. (2) Thermal diffusivity is selected as the key quantity, which is well-defined at the atomistic level. Hence, it is more convincing for the validation of the hydrodynamic description. Also, the dispersion relation focuses on the acoustic collective modes, excluding the physical process of energy and mass transport encoded in the ZFM.

To show the ability of this connection and shed light on some physics remaining undiscovered, here, we focus on the ion-electron nonequilibrium system (electron temperature higher than ion temperature) created by ultrafast laser heating [46-49]. In this condition, the ion dynamics is determined by the optically modulated interatomic potential $[28,50]$, and so are its energy transport and density-density correlation, which serves as an ideal system for understanding the thermally diffusive mode. One should note that only if the large-scale simulations are performed and the connection is constructed can the following results be valid.

\section{DSF AT TWO-TEMPERATURE STATES}

Through DPMD simulations, we calculated the DSF $S(k, \omega)$ of warm dense aluminum at different two-temperature states. Quantitative validation of hydrodynamic description is also performed under these nonequilibrium conditions, as presented in Figs. 1(b) and 1(c). Note that as electron temperature increases, the hydrodynamic regime shifts to lower wave numbers (see Fig. S15 in the Supplemental Material). Considering electron-temperature-influenced interatomic interaction, these results agree with Mithen et al.'s conclusion, indicating that the validity of the hydrodynamic description is related to the characteristic interaction length of the system. Within this extension, we can approach the nearhydrodynamic regime below $0.2 \AA^{-1}$. As shown in Fig. 2(a), at the near-hydrodynamic limit $\left(k=0.097 \AA^{-1}\right)$, the DSF forms the well-known Rayleigh-Brillouin triplet with the central (Rayleigh) peak at zero frequency reflecting thermal relaxation and the two side (Brillouin) peaks coming from acoustic collective excitation that propagates with the adiabatic speed of sound. 

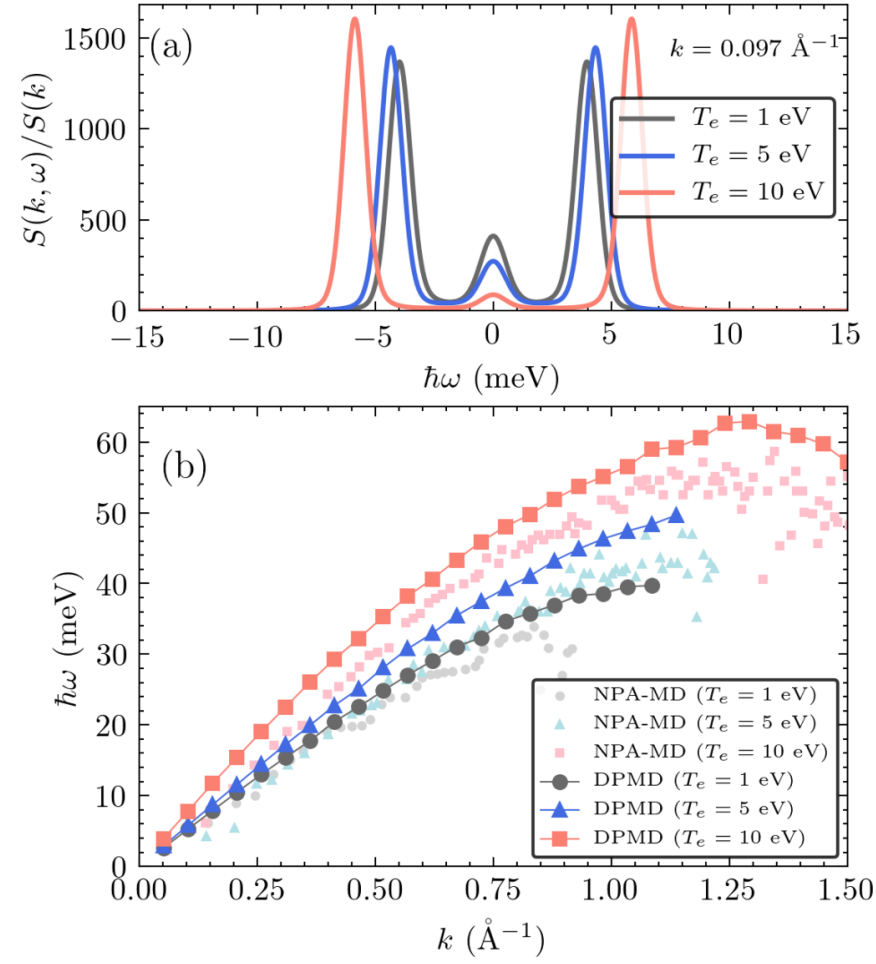

FIG. 2. (a) The DSF for warm dense aluminum is calculated at density $\rho_{0}=2.7 \mathrm{~g} / \mathrm{cm}^{3}$ and ion temperature $T_{i}=1 \mathrm{eV}$ for three different electronic temperatures: $T_{e}=1,5$, and $10 \mathrm{eV}$. For our DPMD simulations, 16384 atoms are used to guarantee that $k=$ $0.097 \AA^{-1}$ is within the confirmed near-hydrodynamic regime below $0.2 \AA^{-1}$. (b) The dispersion relation for two-temperature warm dense aluminum. For comparison, results from NPA-MD simulations are given [18].

Figure 2(a) highlights the significant damping of the ZFM and hardening of the acoustic collective mode at twotemperature states. These trends agree with previous MD results using a neutral pseudoatom (NPA) model [18]. These phenomena can be traced back to the consequence of changed interatomic interactions due to the modification of electron distribution.

In the range of $T_{e}$ studied in this work, from 1 to 10 $\mathrm{eV}$, the mean ionization degree $\bar{Z}$ remained essentially unchanged from the room-temperature value of 3.0 for the normal density of $2.7 \mathrm{~g} / \mathrm{cm}^{3}$ [18,51]. For aluminum, a typical free-electron-like metal with no $d$ bands near the Fermi level, the electron temperature effects originate from the excited electrons, which behave as freelike when $T_{e}$ increases [52]. In this case, the increase in the pressure is related to only an increase in its electronic kinetic part, while the other contributions remain nearly constant. The total pressure rises from 43 to $218 \mathrm{GPa}$ as electron temperature rises from 1 to $10 \mathrm{eV}$. As a result, the ion-ion effective repulsive interaction is strengthened, supported by the fact that the isothermal compressibility $\chi_{T}$ of the ion system decreases from 1.086 to $0.445 \mathrm{eV}^{-1}$. Through the relation $c_{s}^{2}=\gamma /\left(\rho m \chi_{T}\right)$, the enhanced adiabatic sound speed $c_{s}$ can be derived from this lower isothermal compressibility, consistent with the hardening of acoustic modes, as shown in Fig. 2(b). In addition, the
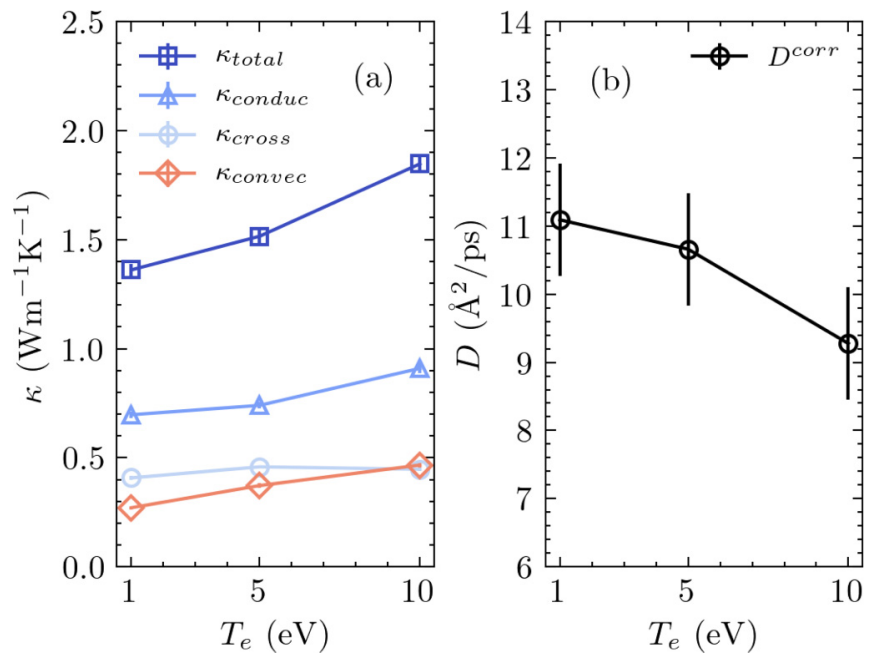

FIG. 3. (a) Thermal conductivities $\kappa$. (b) Diffusion coefficients $D$ of two-temperature warm dense aluminum calculated from DPMD. For the diffusion coefficients, the results were fitted to $D^{\text {corr }}=$ $D^{\text {calc }}+k_{B} T_{i} \xi / 6 \pi \eta L$ for the removal of the size effect, where $D^{\text {calc }}$ is the calculated self-diffusion coefficient via mean-square displacement, $D^{\text {corr }}$ is the corrected value, $L$ is the length of the edge of the cubic simulation box, $k_{B}$ is Boltzmann's constant, $T_{i}$ is the ionic temperature, $\eta$ is the shear viscosity, and $\xi$ is a free parameter for the fitting process.

difference in the dispersion relationship between DP and NPA models emphasizes the importance of considering many-body interaction in the WDM regime $[37,53]$.

\section{HYDRODYNAMIC INSIGHTS INTO THE ATOMIC SCALE}

The damping of the ZFM encodes the change in thermal relaxation processes. Based on the verified connection between $\kappa$ and the ZFM in Figs. 1(b) and 1(c), the strong damping of the ZFM can be considered the enhancement of thermal transport. For a typical dynamic disordered system, ionic energy transport in WDM is contributed by the interatomic interactions and ionic diffusion, corresponding to heat conduction and convection, respectively. To further explore the mechanism of the damping in the ZFM at two-temperature states, we extracted the microscopic dynamics of the energy transport from the expression of the heat current $\mathbf{J}$ [54]. The heat current can be decomposed into a heat conduction term and a heat convection term; thus, the total thermal conductivity can be decomposed into the contributions from the conduction term $\kappa_{\text {conduc }}$, the convection term $\kappa_{\text {convec }}$, and the cross term $\kappa_{\text {cross }}$.

The first term, $\kappa_{\text {conduc }}$, is contributed by the interatomic interaction. Because of the ill-defined phonons, collective excitations are the main heat carriers in WDM. As a result of increased electron temperature, the strengthened ion-ion interaction causes hardening of the ion longitudinal collective mode. These acousticlike excitations usually contribute overwhelmingly to heat conduction in amorphous materials [55]. Therefore, the increase in group velocity directly leads to increased heat conduction [Fig. 3(a) and thus the damping of the ZFM. 
The second term, $\kappa_{\text {convec }}$, results from the convection of particles, which accounts for the heat carried by ion diffusional motion. As shown by the calculated diffusion coefficient and thermal conductivity in Fig. 3, the situation becomes more complicated since the mass transport and energy transport show opposite trends under nonequilibrium conditions. The diffusion coefficient describes the ability of ions to leave the local trap $[56,57]$. In the strong-coupling regime, the strong interaction gives rise to the cage effect, whereby each particle finds itself trapped for some period of time until it overcomes the energy barrier and diffuses to a neighboring cage $[53,58]$. From Fig. 3(b) we can see the diffusion coefficients decrease with respect to the increase of electronic temperature for two-temperature states. The backscattering of ions due to collisions with nearest neighbors plays significant roles at elevated electron temperature, making it more difficult for ions to leave the local environments. This physics suppresses single-particle dynamics (diffusional motion) and has a positive contribution to the ZFM in the DSF, causing the increase in the height of the central peak.

Meanwhile, the energy carried by a particle is increased due to elevated electronic temperature under the optical excitation condition. As a consequence of the competition between reduced ionic mass transport and increased energy carried by each particle, the heat convection term of $\kappa$ is increased [Fig. 3(a)], indicating increased energy transport. The last term, $\kappa_{\text {cross }}$, results from the cross correlation between the heat carried by ion diffusion and the ion collective modes, and it exhibits relatively nonsignificant increasing trends compared with $\kappa_{\text {conduc }}$ and $\kappa_{\text {convec }}$ under two-temperature states.

Combining the contributions from collective excitation and ionic diffusional motion, we obtained a damped central peak in two-temperature warm dense aluminum (known as an enhanced thermally diffusional mode). From this perspective, we can know that the coupling of single-particle diffusion and collective dynamics determines the shape of the ZFM. In addition, it is worth clarifying the ambiguities between thermal diffusion and mass self-diffusion. Their distinct behaviors under nonequilibrium conditions suggest that one cannot simply attribute the central peak to the random collision between ions because the ZFM is also associated with temperature fluctuations.

\section{SUMMARY}

Beyond the conventional framework of ion modes (densitydensity correlation), we combined the microscopic and hydrodynamic descriptions of the ionic DSF $[1,19]$ to have a clear discussion of the physical origin of the relaxation process revealed by the DSF. With the generated DP, large systems and long trajectories are accessible to capture the ion dynamics near the hydrodynamic limit with ab initio accuracy. Choosing thermal diffusivity as a bridging medium, we quantitatively verified the relationship between the ZFM in the DSF and energy transport. Specifically, as the spatial scale approaches the hydrodynamic regime, the thermal diffusivity obtained from the DSF gradually converges to the benchmark value. This result bridges the atomic scale and hydrodynamic description. Transport properties like thermal conductivity can be extracted from direct measurement of the ion-ion DSF at small $k$ values. Most importantly, as the hydrodynamic description points out, the relaxational behavior of the ZFM originates from the macroscopic thermal process (energy transport), shedding light on the understanding of the ion modes in the WDM regime. Based on a verified relationship, we presented specific analysis of the microscopic mechanism behind the damping of the ZFM for nonequilibrium warm dense aluminum, and a competitive mechanism between hardening of acoustic collective excitations and suppression of ionic self-diffusion was found. The ZFM reveals the coupling of single-particle diffusional motion and ionic collective motion; it would be helpful to understand the strongly enhanced central peak in the DSF induced by the nonadiabatic effect $[6,59,60]$.

\section{ACKNOWLEDGMENTS}

The authors thank Prof. J. M. Yuan for his insightful discussion. This work was supported by the National Key R\&D Program of China under Grant No. 2017YFA0403200, the National Natural Science Foundation of China under Grants No. 11774429, No. 11874424, and No. 12047561, the NSAF under Grant No. U1830206, the Science Challenge Project under Grant No. TZ2016001, and the Science and Technology Innovation Program of Hunan Province under Grant No. 2020RC2038.
[1] J.-P. Hansen and I. R. McDonald, Theory of Simple Liquids (Elsevier Academic Press, London, 2006).

[2] B. Lu, D. Kang, D. Wang, T. Gao, and J. Dai, Chin. Phys. Lett. 36, 103102 (2019).

[3] M. W. C. Dharma-wardana, D. D. Klug, and R. C. Remsing, Phys. Rev. Lett. 125, 075702 (2020).

[4] T. Bryk and I. Mryglod, J. Phys.: Condens. Matter 13, 1343 (2001).

[5] V. M. Giordano and G. Monaco, Proc. Natl. Acad. Sci. U.S.A. 107, 21985 (2010).

[6] P. Mabey, S. Richardson, T. G. White, L. B. Fletcher, S. H. Glenzer, N. J. Hartley, J. Vorberger, D. O. Gericke, and G. Gregori, Nat. Commun. 8, 14125 (2017).

[7] T. G. White, S. Richardson, B. J. B. Crowley, L. K. Pattison, J. W. O. Harris, and G. Gregori, Phys. Rev. Lett. 111, 175002 (2013).
[8] H. R. Ruter and R. Redmer, Phys. Rev. Lett. 112, 145007 (2014).

[9] B. B. L. Witte, M. Shihab, S. H. Glenzer, and R. Redmer, Phys. Rev. B 95, 144105 (2017).

[10] T. Scopigno, U. Balucani, A. Cunsolo, C. Masciovecchio, G. Ruocco, and F. Sette, Philos. Mag. B 79, 2027 (1999).

[11] T. Scopigno, G. Ruocco, and F. Sette, Rev. Mod. Phys. 77, 881 (2005).

[12] S. Hosokawa, M. Inui, Y. Kajihara, K. Matsuda, T. Ichitsubo, W. C. Pilgrim, H. Sinn, L. E. Gonzalez, D. J. Gonzalez, S. Tsutsui, and A. Q. R. Baron, Phys. Rev. Lett. 102, 105502 (2009).

[13] T. Guillot, Science 286, 72 (1999).

[14] P. Lorazo, L. J. Lewis, and M. Meunier, Phys. Rev. Lett. 91, 225502 (2003).

[15] J. Lindl, Phys. Plasmas 2, 3933 (1995). 
[16] S. Ichimaru, Rev. Mod. Phys. 54, 1017 (1982).

[17] J. Vorberger, Z. Donko, I. M. Tkachenko, and D. O. Gericke, Phys. Rev. Lett. 109, 225001 (2012).

[18] L. Harbour, G. D. Forster, M. W. C. Dharma-wardana, and L. J. Lewis, Phys. Rev. E 97, 043210 (2018).

[19] J. P. Mithen, J. Daligault, and G. Gregori, Phys. Rev. E 83, 015401(R) (2011).

[20] J. P. Mithen, J. Daligault, B. J. B. Crowley, and G. Gregori, Phys. Rev. E 84, 046401 (2011).

[21] D. Kang and J. Dai, J. Phys.: Condens. Matter 30, 073002 (2018).

[22] S. H. Glenzer and R. Redmer, Rev. Mod. Phys. 81, 1625 (2009).

[23] L. B. Fletcher et al., Nat. Photonics 9, 274 (2015).

[24] L. Zhang, J. Han, H. Wang, R. Car, and W. E, Phys. Rev. Lett. 120, 143001 (2018).

[25] H. Wang, L. Zhang, J. Han, and W. E, Comput. Phys. Commun. 228, 178 (2018).

[26] L. Zhang, D.-Y. Lin, H. Wang, R. Car, and E. Weinan, Phys. Rev. Mater. 3, 023804 (2019).

[27] Y. Zhang, C. Gao, Q. Liu, L. Zhang, H. Wang, and M. Chen, Phys. Plasmas 27, 122704 (2020).

[28] V. Recoules, J. Clérouin, G. Zérah, P. M. Anglade, and S. Mazevet, Phys. Rev. Lett. 96, 055503 (2006).

[29] P. Giannozzi et al., J. Phys.: Condens. Matter 29, 465901 (2017).

[30] J. P. Perdew, K. Burke, and M. Ernzerhof, Phys. Rev. Lett. 77, 3865 (1996).

[31] P. E. Blöchl, Phys. Rev. B 50, 17953 (1994).

[32] N. Holzwarth, A. Tackett, and G. Matthews, Comput. Phys. Commun. 135, 329 (2001).

[33] A. Baldereschi, Phys. Rev. B 7, 5212 (1973).

[34] S. Plimpton, J. Comput. Phys. 117, 1 (1995).

[35] S. Nosé, J. Chem. Phys. 81, 511 (1984).

[36] W. G. Hoover, Phys. Rev. A 31, 1695 (1985).

[37] See Supplemental Material at http://link.aps.org/supplemental/ 10.1103/PhysRevResearch.3.033116 for details about KS-DFT simulations, DP training, DPMD simulations, verifying the ability of DP, and fitting DSF to the hydrodynamic description.

[38] Q. Liu, D. Lu, and M. Chen, J. Phys.: Condens. Matter 32, 144002 (2020).

[39] B. Cheng and D. Frenkel, Phys. Rev. Lett. 125, 130602 (2020).
[40] M. S. Green, J. Chem. Phys. 22, 398 (1954).

[41] R. Kubo, J. Phys. Soc. Jpn. 12, 570 (1957).

[42] X. Yu, D. Ma, C. Deng, X. Wan, M. An, H. Meng, X. Li, X. Huang, and N. Yang, Chin. Phys. Lett. 38, 014401 (2021).

[43] D. A. McQuarrie, Statistical Mechanics (University Science Books, Sausalito, 1965).

[44] Z. Wang, S. Safarkhani, G. Lin, and X. Ruan, Int. J. Heat Mass Transf. 112, 267 (2017)

[45] H. Kählert, Phys. Rev. Research 2, 033287 (2020).

[46] R. Ernstorfer, M. Harb, C. T. Hebeisen, G. Sciaini, T. Dartigalongue, and R. D. Miller, Science 323, 1033 (2009).

[47] M. Mo, Z. Chen, R. Li, M. Dunning, B. Witte, J. Baldwin, L. Fletcher, J. Kim, A. Ng, R. Redmer, A. H. Reid, P. Shekhar, X. Z. Shen, M. Shen, K. Sokolowski-Tinten, Y. Y. Tsui, Y. Q. Wang, Q. Zheng, X. J. Wang, and S. H. Glenzer, Science 360, 1451 (2018).

[48] Z. Chen, M. Mo, L. Soulard, V. Recoules, P. Hering, Y. Y. Tsui, S. H. Glenzer, and A. Ng, Phys. Rev. Lett. 121, 075002 (2018).

[49] Q. Zeng and J. Dai, Sci. China: Phys., Mech. Astron. 63, 1 (2020).

[50] Q. Ma, J. Dai, D. Kang, M. S. Murillo, Y. Hou, Z. Zhao, and J. Yuan, Phys. Rev. Lett. 122, 015001 (2019).

[51] Y. Hou, Y. Fu, R. Bredow, D. Kang, R. Redmer, and J. Yuan, High Energy Density Phys. 22, 21 (2017).

[52] F. Bottin and G. Zérah, Phys. Rev. B 75, 174114 (2007).

[53] Y. Hou, J. Dai, D. Kang, W. Ma, and J. Yuan, Phys. Plasmas 22, 022711 (2015).

[54] X. Yu, R. Li, T. Shiga, L. Feng, M. An, L. Zhang, J. Shiomi, and N. Yang, J. Phys. Chem. C 123, 26735 (2019).

[55] F. DeAngelis, M. G. Muraleedharan, J. Moon, H. R. Seyf, A. J. Minnich, A. J. H. McGaughey, and A. Henry, Nanoscale Microscale Thermophys. Eng. 23, 81 (2019).

[56] J. Dai, Y. Hou, D. Kang, H. Sun, J. Wu, and J. Yuan, New J. Phys. 15, 045003 (2013).

[57] H. Y. Sun, D. Kang, Y. Hou, and J. Y. Dai, Matter Radiat. Extremes 2, 287 (2017).

[58] J. Daligault, Phys. Rev. Lett. 96, 065003 (2006).

[59] B. Larder, D. O. Gericke, S. Richardson, P. Mabey, T. G. White, and G. Gregori, Sci. Adv. 5, eaaw1634 (2019).

[60] R. A. Davis, W. A. Angermeier, R. K. T. Hermsmeier, and T. G. White, Phys. Rev. Research 2, 043139 (2020). 